\title{
Switching of lasing wavelength and threshold current of Semiconductor Laser by temperature Variation
}

\author{
Suraj Manikandan \\ Department Of Photonics \\ Ghent University \\ Belgium \\ suraj.manikandan@ugent.be
}

\begin{abstract}
The present work is theoretical and experimental study of temperature effect on wavelength and threshold current. Since Semiconductor lasers are the type of lasers which uses semiconductor material as a gain medium to achieve stimulated emission of radiation. In this module, the type of semiconductor lasers use is VCSEL and laser diode. Temperature change cause Semiconductor lasers to shift its threshold current, this variation also causes a shift in output wavelength. The experimental results highly agreement with the theoretical calculations.
\end{abstract}

Keyword: Temperature effect, VCSEL, Laser Diode, Wavelength Shift, Band gap energy, Radiative Absorption, stimulated emission

\section{INTRODUCTION}

A semiconductor laser is also known as laser diode in construction wise its similar to LED, which converts electrical energy to light energy directly. The only difference in working, gain in the semiconductor is created due to pumping. Without pumping most of the electron will be valance band A pump beam with photon energy slightly above the bandgap energy can excite electrons into a higher state in the conduction band, from where they quickly decay to states near the bottom of the conduction band. So due to this property, it has a longer lifetime, if they are handled properly with the proper driver circuit. There is the number of reason for the laser to fail is current surge typically $9 \mathrm{~mA}$ for VCSEL and $80 \mathrm{~mA}$ for Laser diode, electrostatic surge and temperature surge. Temperature is the best way to characterize the sensitivity of laser efficiently. Thermal characterization gives an insight into the device functionality and a point for better laser design in transition level. For laser diode particularly most critical parameter like wavelength, threshold current depends strongly on temperature.

The concept of heat generation in laser and transportation of this heat from the laser is one of the important aspects of laser design through this understanding one can change the lasing wavelength of the laser in biomedical application. An important question is how can temperature in the laser diode is increased basically by increasing the injection current through the resistive layer and laser is generated by process of radiative absorption and non-radiative recombination due to which light is emitted rather than heat. Increasing temperature will lead to a large spread in the energy of carrier distribution in the active layer consequence is a decrease in efficiency.

\section{Calculation for EFFect of VARiation OF}

TEMPERATURE ON THE THRESHOLD CURRENT AND

WAVELENGTH ON OUTPUT OF SEMICONDUCTOR LASER

\section{A. Effect of Temperature On The Threshold Current}

For Current, a semiconductor laser to work or operate gain must be higher than the loss in laser, and the point is called threshold. So current at a point where the gain is equal to losses is called threshold current. Since power characteristics of laser strongly depend on temperature because of the following reason:

1. In active region recombination of holes and electron

2. Enhancement of Non radiative recombination

The threshold current dependant on temperature in exponential, due to this relation

$$
I\left(T_{2}\right)=I_{t}\left(T_{1}\right) \exp \left[\left(T_{2}-T_{1}\right) / T_{0}\right]
$$

Where $I_{t}\left(T_{1}\right)$ and $I_{t}\left(T_{2}\right)$ are the threshold current in tem-

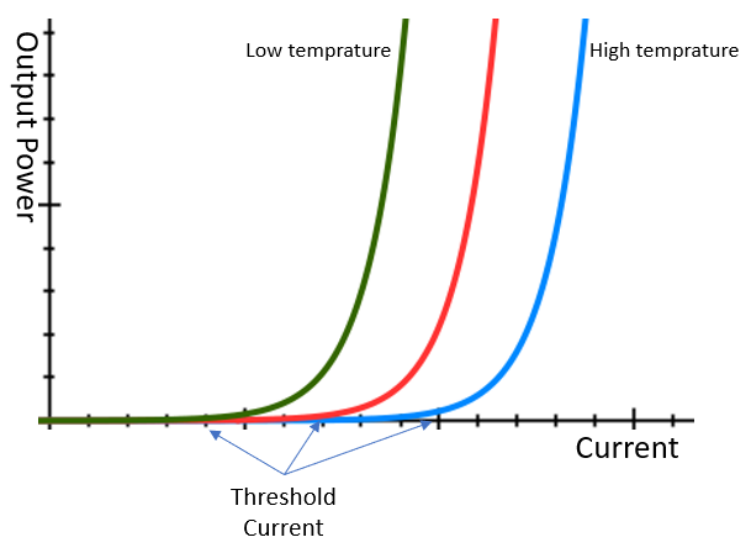

Fig. 1. Light output vs laser bias current, showing the threshold current

perature $T_{1}$ and $T_{2}$ and $T_{0}$ is scaling factor whose typical 
value is $150 \mathrm{k}$. Example calculation assume if $T_{1}=0 C^{\circ}$ and $T_{2}=40 C^{o}$, now threshold current will be 28 percent high at high temperature.

$$
P=Z\left(I-I_{t h}\right)
$$

Where $\mathrm{P}$ is the output power and $\mathrm{Z}$ is a constant with value $1.21 \mathrm{~W} / \mathrm{A}$. I is supply current and $I_{t h}$ is threshold current of laser diode.

\section{B. Effect of temperature on Wavelength Shift}

The emissions wavelength of a semiconductor depend on the amount of energy is released in the form of a photon during recombination of hole and electron this energy released is also known as band gap energy of semiconductor. Control of temperate is very essential for the lasing wavelength emission. Wavelength tuning of a laser can be done many ways such as varying diode current, varying heat sink temperature using Peltier plate and last is applying a magnetic field. During these methods, there is a change in injection current concentration in the semiconductor which ultimately change the refractive index of the cavity and it also shifts the peak photon energy.

$$
\lambda_{(T)}=\lambda_{0}+K_{\lambda}\left(T_{j}-300 K\right)
$$

Where $K_{\lambda}$ is temperature coefficient and $\lambda_{0}$ is wavelength at $300 \mathrm{~K}$

\section{EXPERIMENTAL MEASUREMENTS}

The experiment setup is consist of semiconductor lasers such as Laser diode and Vertical Cavity Surface Emitting Lasers (VCSELs) with a power meter and Spectrum Analyser. Power meter uses is a Newport power meter This detector consists of two parts: a measuring unit and a display unit. The measuring unit contains the semiconductor material to which the light should be guided. A spectrum analyser measures the intensity of the light at different wavelengths. During the lab sessions, you will use a fibre optic spectrometer. More, in particular, the Avaspec-3648-USB2 from Avantes.

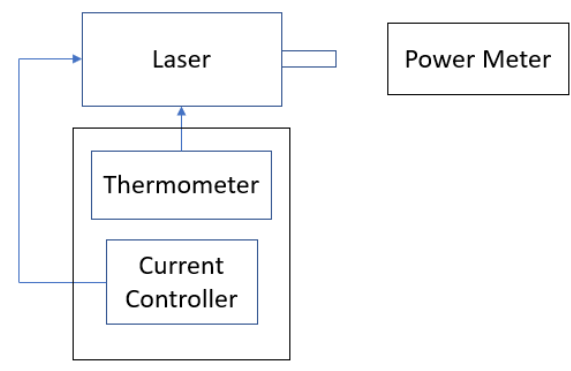

Fig. 2. Experiment is used for measuring the threshold current for laser diode

\section{A. Temperature VS Threshold Current}

In this experiment, laser setup is connected to power meter for the measurement of output power with the variation of injection current which is been controlled by a current controller with a thermometer. There are two types of a semiconductor laser has been used such as laser diode and Vertical Cavity Surface Emitting Lasers (VCSELs) with the different current rating. For VCSEL current should be varied between 0 to $9 \mathrm{~mA}$ and laser diode has a more current rating so current is varied between 0 to $90 \mathrm{~mA}$. Since output power depends on temperature so the temperature is maintained constant during reading values. Set of reading is repeated for different temperature and for both semiconductor lasers. Finally, plot the current vs output power for different temperature(15C $35 \mathrm{C}$ ) and it clearly shows the threshold current is got increased for increased in temperature.

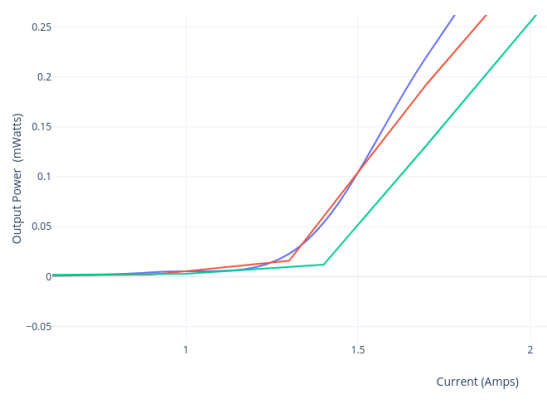

Fig. 3. Variation of the Light output in function of temperature for laser diode

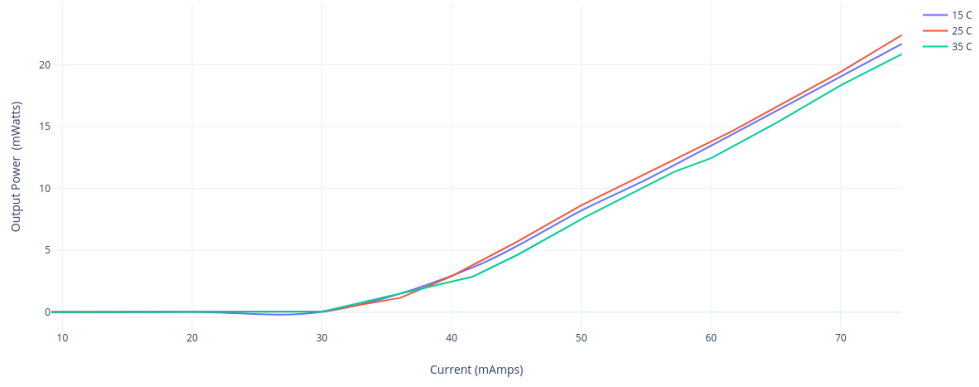

Fig. 4. Variation of the Light output in function of temperature for VCSEL

\section{B. Temperature VS Emitted Wavelength}

In this part of experiment output of the lasers is connected to spectrum analyzer for the the measurement of wavelength is been emitted. For the Mitsubishi laser diode, the spectral distribution is measured when the temperature is xed at $25 \mathrm{C}$ and the input current is set at three dierent values. The other way around, the input current xed is at $50 \mathrm{~mA}$ and then the temperature is respectively $15 \mathrm{C}, 25 \mathrm{C}$ and $35 \mathrm{C}$.One can see that the power intensity peak shifts to higher wavelengths 
when the input current increases in case of xed temperature. The same goes for when the temperature increases at a xed input current. When the temperature goes up, the band gap decreases resulting in a peak shift to longer wavelengths. When the input current is increased, the optical path length changes which also results in a peak shift.

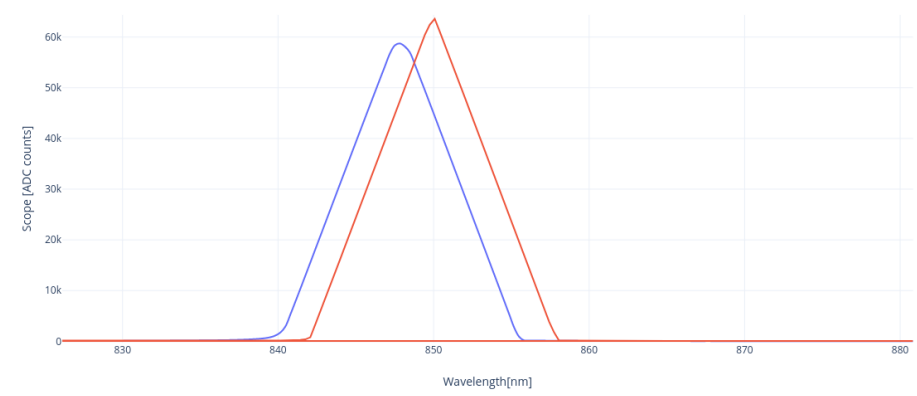

Fig. 5. Variation of the shifted wavelength of laser diode as a function of the temperature for VCSEL

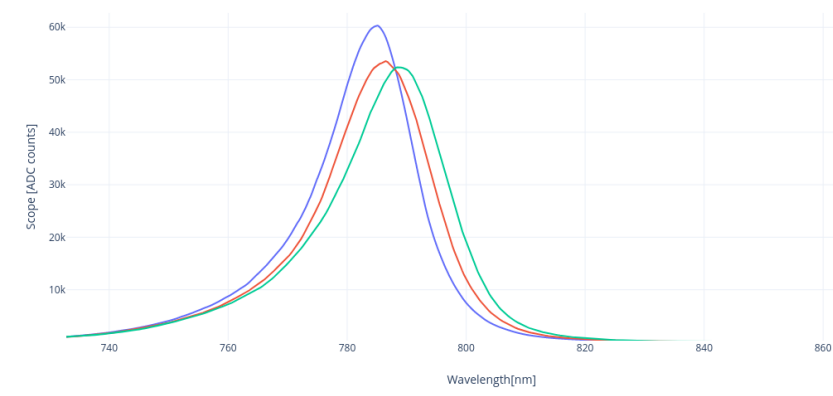

Fig. 6. Variation of the shifted wavelength of laser diode as a function of the temperature for Laserdiode

\section{CONCLUSION}

A semiconductor laser's emitted wavelength is a temperature-dependent amount, increasing as temperature rises. This is consistent with previous research and calculated expectations. An experimental study for effect varies temperatures on Semiconductor Laser's shifting wavelength, experimental results of wavelength vary range (785nm-795 $\mathrm{nm})$, temperature change from $(15-35 \mathrm{C})$, rate change $(0.25$ $0.3 \mathrm{~nm} / \mathrm{C})$. The empirical, theoretical impact findings vary temperatures that match about 95 percent of Semiconductor Laser's moving wavelength.

\section{REFERENCES}

[1] Yi, H. J. (1995), Temperature dependence of threshold current density Appl. Phys. Lett., 66(3):25355.
[2] Yang,R. Q. , Cory J. Hill, Kamjou Mansour, Yueming Qiu, Alexander Soibel, Richard E. Muller, and Pierre M. Echternach, (2007) Distributed Feedback Mid-IR Interband Cascade Lasers at Thermoelectric Cooler Temperatures, Springer- Verlag, IEEE Journal, VOL. 13, NO. 5 , September/October.

[3] Albers, P.(2009), Semiconductor Laser catalog, CRC Press, Taylor and Francis Group, Florida. ISBN 978-1-4665-0990-0.

[4] Johnson,L.A.(2003), )Controlling Temperatures of Diode Lasers Thermoelectrically, ILX Light wave Corporation. 